\title{
Topology optimization and additive manufacturing of an optical housing for space applications
}

\author{
Nils Heidler ${ }^{1 *}$, Henrik von Lukowicz ${ }^{1,2}$, Enrico Hilpert ${ }^{1,2}$, Stefan Risse $^{1}$, Lucas $_{\text {Alber }}{ }^{3}$, Jan Klement ${ }^{3}$, Frank Heine ${ }^{3}$, \\ Ralf Bölter $^{3}$ and Josep Maria Perdigues Armengol ${ }^{4}$ \\ ${ }^{1}$ Fraunhofer Institute for Applied Optics and Precision Engineering IOF, 07745 Jena, Germany \\ ${ }^{2}$ Institute of Applied Physics, Friedrich-Schiller-University, 07745 Jena, Germany \\ ${ }^{3}$ Tesat-Spacecom GmbH \& Co. KG, 71522 Backnang, Germany \\ ${ }^{4}$ ESA/ESTEC, 2200 AG Noordwijk ZH, Netherlands
}

\begin{abstract}
The design of an optical housing for laser telecommunication in space is improved by topology optimization. Different mechanical and thermal boundary conditions are considered while minimizing the overall weight of the housing. As a proof-of-concept study, a complex and lightweight housing is made by additive manufacturing with the aluminium silicon alloy AlSi40. Post processing steps include a thermal treatment, cleaning and a mechanical machining process. Final characterization tests include the evaluation of material characteristics by tensile tests, a computed tomography scan and a CMM measurement. The final shock and vibrational test is used to proof the performance of the housing for future space applications.
\end{abstract}

\section{Introduction}

Opto-mechanical structures used in aerospace applications usually have to meet challenging and partially contradicting requirements. This study aims to demonstrate exemplarily that topological optimization combined with 3D printing can successfully decrease the weight of an opto-mechanical housing inside a laser communication terminal while keeping the existing requirements concerning thermal conductivity and mechanical stability.

Conventional machining processes restrict the possible design of housings, where the high freedom of design of additive manufacturing processes offer new concepts for optimized components $[1,2]$.

The initial design of the housing is made out of AlBeMet, a beryllium and aluminium metal matrix composite material. This material offers very good mechanical properties but due to its carcinogenic properties, the production process involves difficulties and risks. As alternate material, the aluminium silicon alloy AlSi40 with 40 weight percent silicon offers good mechanical properties and the coefficient of thermal expansion is close to AlBeMet [3]. This avoids a thermoelastic redesign of the whole opto-mechanic system.

The optimization process aims on a lightweight design with additional mechanical and thermal boundary conditions. Starting with an envelope model of the housing, different topology optimized model variants arise. Based on the overall performance, the best model variant is processed by SLM. Afterwards, thermal as well as mechanical post processing steps and metrological characterizations of the housing finalize the study.

\section{Topology Optimization}

The basis of the topology optimization is the envelope model of the optical housing, defining the necessary mechanical mounting interfaces for the optical components and other structural parts, see Fig.1. Within the volume of the envelope, the simulation program ANSYS is allowed to alter the material distribution to optimize the design. The applied boundary conditions involve the overall mass, geometrical, mechanical and thermal constraints. Load cases for quasi-static acceleration, sinus vibration, random vibration, shock, eigen-frequencies and static and transient thermal constraints are given. After one optimization step, all load cases are verified based on FEM simulations of the obtained design variant. The final evaluation is done by verifying if all initial conditions are met.
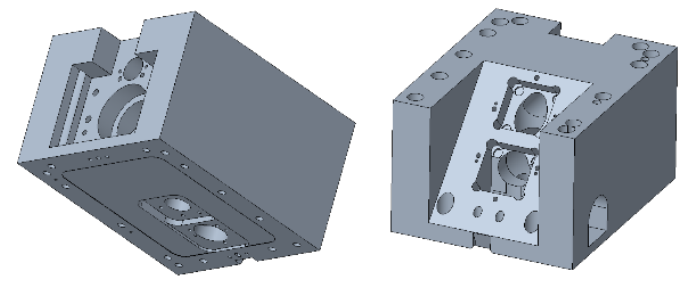

Fig. 1. CAD illustration of envelope model.

As exemplarily result, the first natural eigen-frequency of the final design variant occur at approx. $2850 \mathrm{~Hz}$, see Fig. 2 . This is well above the specified minimum frequency and above the typical incitation range for random vibration. The other given specifications are met as well.

\footnotetext{
* Corresponding author: Nils.Heidler@iof.fraunhofer.de
} 


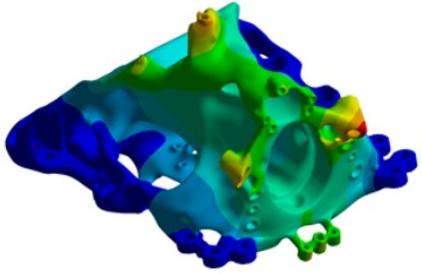

Fig. 2. Result of FEM simulation (modeshape of first eigenfrequency at $2850 \mathrm{~Hz}$ ) of the optimized model.

\section{Additive Manufacturing}

The preparation of the optimized model for the AM process involves the addition of allowance material. This is necessary to provide enough material for the mechanical machining of the functional surfaces during the post processing steps. Using the software "Materialise Magics", the orientation of the CAD model within the building volume of the AM machine is set, supporting structure for the mechanical and thermal connection to the baseplate is defined and finally the slicing of the complete model into thin layers is accomplished [4].

For the additive manufacturing of the model, a powder-bed based, selective laser melting process is used. As AM machine, the Concept Laser M2 uses a fibre laser source with up to $400 \mathrm{~W}$ power and a heatable building chamber. Spherical aluminium silicon powder (AlSi40) with diameters between $20 \mu \mathrm{m}$ and $60 \mu \mathrm{m}$ is processed under protective gas. The used process parameters are feasible to generate volume parts with a low porosity $<0.05 \%$ [4]. Fig. 3 shows the optimized model after the finished AM process. Additional tensile specimens are manufactured to facilitate material tests.

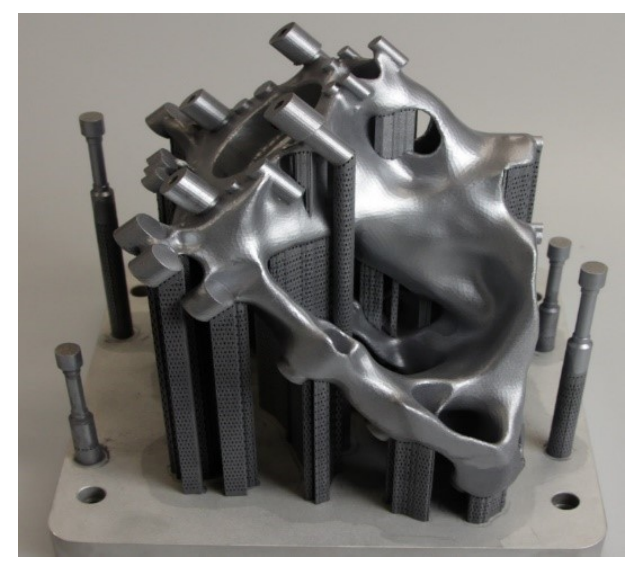

Fig. 3. Optical housing and tensile specimens made of AlSi40 after the AM process.

\section{Post Processing and Characterization}

After the AM process, the whole building plate including all parts is thermal treated to minimize the residual internal stress. An ultrasonic cleaning step is applied to remove the adhering powder particles from the surface. No further surface modification such as sandblasting or polishing are used. The optical housing is finished on a 5-axis milling machine by removing the allowance material and adding tapped holes and holes with fitting tolerances, see Fig. 4. These features cannot be realized during the AM process due to the typical surface roughness between $5 \mu \mathrm{m}$ RMS and $20 \mu \mathrm{m}$ RMS. Tactile measurements on a coordinate measurement machine are used to control the correct position of all machined interfaces.

A computed tomography (CT) scan is performed for quality assurance. No defects or pores are visible within the resulting voxel resolution of $120 \mu \mathrm{m}$.

The tensile test results reveal the good mechanical properties of the AlSi40 material with a tensile strength of approx. $260 \mathrm{MPa}$ and a Young's Modulus of approx. 100 GPa. Compared to conventional AlSi40 base material the increased tensile strength of AM made AlSi40 can be explained by the finer grain structure of the material [5].

A shock and vibration test is planned to verify the FEM simulations.

\section{Conclusion and Outlook}

This study presents the topology optimization of an optical housing for space communication and the additive manufacturing of the resulting design out of AlSi40. All thermal and mechanical specification have been fulfilled and the overall weight was reduced by $20 \%$ compared to the initial AlBeMet housing. A CT scan, tensile tests and a shock and vibration test, verifies the good properties of the housing. Further material test shall verify the stability of the process and its application for future space missions.

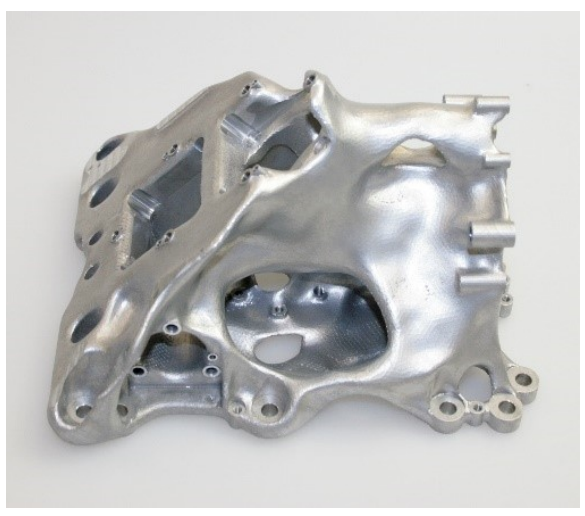

Fig. 4. Topology optimized optical housing, made by AM out of AlSi40

\section{References:}

1. Patent WO2013/017144 A1, Method for manufacturing a mirror comprising at least one cavity and optical mirror

2. E. Brandl, U. Heckenberger, V.Holzinger, D.Buchbinder, Mat.\&Des. 34, p. 159-169, (2012)

3. J. Kinast, K. Grabowski, A. Gebhardt, R.R. Rohloff, S. Risse, A. Tünnermann, Proc. of SPIE Vol. 10563, (2014)

4. E. Hilpert, J. Hartung, H.v. Lukowicz, T. Herffurth, N. Heidler, Optical Engineering (to be published)

5. E. Hilpert, S. Risse, MS\&T (2015) 